\title{
Special Issue on Intelligent Support for Learning in Groups
}

\author{
Rohit Kumar • Jihie Kim
}

Published online: 27 December 2013

(C) International Artificial Intelligence in Education Society 2013

\section{Introduction}

Advances in Intelligent Tutoring Systems (ITS) over the past two decades have enabled the development and deployment of highly effective learning technologies that support learners across a wide-range of domains and age-groups. On the other hand, research in collaborative learning has informed instructional approaches that leverage the pedagogical benefits of learning in groups (including dyads, small groups and teams). The use of these approaches in digital environments is being actively studied in the field of computer supported collaborative learning (CSCL).

In addition to the increased use of computing devices in traditional classrooms, technological advancements such as mass access to revolutionary communication technologies have prompted the adoption of web-based tools for educational purposes. Educational service providers are deploying web-based platforms that allow students to access resources that supplement classroom instruction and allow students to share their learning experience with their peers. Hundreds of hours of high quality learning content and thousands of educational games that enrich learning interactions are accessible through mobile/tablet devices.

Harmonious use of these resources and scientific developments offer opportunities for breaking new ground in the application of artificial intelligence (AI) technologies for education. Techniques developed in the field of AI for Education (AIEd) such as student/tutor/domain modelling, integrated assessment, tutorial dialog, automated scaffolding, data mining and pedagogical agents can further improve the effectiveness of learning in groups. On the other hand, multi-party interaction and massive online learning platforms present AIEd researchers with exciting next generation challenges.

The IJAIEd Special Issue on Intelligent Support for Learning in Groups (ISLG) includes four articles that give us a brief peek at recent advances in some of these

\footnotetext{
R. Kumar $(\triangle)$

Raytheon BBN Technologies, Speech, Language and Multimedia Department, Cambridge, MA, USA

e-mail: rkumar@bbn.com

J. $\operatorname{Kim}(\bowtie)$

Information Sciences Institute, University of Southern California, Los Angeles, CA, USA

e-mail: jihie@isi.edu
} 
research directions. In this preface, we will characterize ISLG as a field of interest within AIEd and elaborate on the four dimensions that define the scope of this field. We will present editorial comments on the articles included in this issue followed by an outlook on the growing field of ISLG.

\section{Intelligent Support for Learning in Groups}

The field of ISLG is defined by the four alphabets that make up the abbreviation. Foremost, Intelligent technologies are at the heart of differentiating this field from work conducted by CSCL researchers. Second, work within this field is targeted towards supporting one or more stakeholders or learning and teaching processes. Third, the focus on learning applications differentiates this field from research in broader application of intelligent technologies. Finally, it is important to identify the characteristics of group to situate work in ISLG.

While at first look, it may be seem that the scope of this field is extremely narrow, due to the constraints of combining these four dimensions, it is in fact the contrary. The plethora of techniques and aspects that researchers focusing on each of these dimension are exploring can combine into exciting new applications, interesting questions and corresponding research directions. In this rest of this section, we will elaborate on aspects of each of these dimensions as well as bring attention to some of the prior and recent publications leading up to this special issue.

\section{Intelligent Technologies}

The entire breadth of Intelligent Technologies, ranging from classical AI techniques such as planning and knowledge representation to contemporary approaches such as statistical machine learning, are applicable to ISLG. Widely successful AIEd techniques such as student modelling, example tracing and discourse analysis are already being applied to ISLG publications in the two recent ISLG workshops (Kumar and Kim 2013; Kim and Kumar 2012) that led up to this special issue. Other techniques discussed in the papers presented at these workshops included sentiment analysis, conversational agents, expert knowledge bases, Bayesian models, social recommendation and open learning models (OLMs). Note that, allied AIEd fields such as Educational Data Mining (EDM) share common interests with ISLG on this dimension.

\section{Support Offered}

One of the ways to characterize the forms of support offered to learning in groups is based on identifying the stakeholder(s) in the learning process being supported. In different instantiations of learning groups, some of the stakeholders include learners, teachers, mentors, content creators, administrators and researchers. Each of the various common forms of support such as interactive feedback, assessment, engagement, content recommendation, customizability and authoring/development tools are applicable to one or more of these stakeholders. Existing educational tools and support can be revisited in the context of ISLG. The use of conventional forms of personalized support for group activities brings attention to aspects such as the types of individual 
contributions that should be captured and monitored in the context of group learning, the distribution of learning tasks among the individuals in a group and the transfer of effective strategies across groups.

\section{Learning Applications}

Group learning applications can be characterized by many aspects that situate the learning. Foremost, the learning domains where group learning has been applied range from conventional subjects such as mathematics, science, languages and programming to innovative domains designed to incite curiosity such as urban planning, public policy and critical reasoning. In addition to domains common to grade-school and college curriculum, applications in team training, professional development and lifelong learning are likely to be explored within the field of ISLG. Applications can also be characterized by the learning environments and the structure of learning activity. In addition to real-world environments like classrooms, a variety of virtual environments with different interactive affordances (such as synchronous/asynchronous, text/speech, immersive, etc.) have been used. Growing interest in massive open online courses (MOOCs) offers an opportunity to apply ISLG to learning environments that maybe widely adopted. The characterization of the learning application is critical to describing work in ISLG to understand its generalizability.

\section{Group Characteristics}

Learning groups exists in many shapes and sizes. A group can be as small as a dyad and as large as a community. The characteristics of the group are closely tied to those of the learning application. Additionally, the composition of the groups can range from set of individuals with homogenous characteristics, to participants that have various roles assigned to them (e.g. peer, tutor, mentor, observer, teacher) and have varying skills and capabilities (e.g. expert, novice). The groups may be fixed during the course of the learning or maybe constantly evolving. In either case, their construction maybe ad-hoc or carefully crafted. ISLG researchers take into consideration these characteristics while choosing the intelligent technologies and supports offered.

While defining the scope of ISLG, it is important to bring attention to some of prior work that is closely related to the field of ISLG including key publications in this journal. Group learning theories are rooted in Vygotsky's (1978) notion of learning through social development. Similarly rooted, situated learning theory was proposed as a model of learning in a community of practice with focus on learning by doing, and addressing real problems (Lave and Wenger 1991). Researchers have investigated learning benefits from collaborative learning, inquiry and discovery oriented activities, and reflective interaction among students (Bransford et al. 2000; Felder 1995). Within the AIEd community, over two decades back, Baker (1994), applied discourse analysis techniques to model negotiation interaction in dyads. Similarly, Barros and Verdejo (2000) analysed group interaction to model learning processes. This work introduces the use of group activity for assessment and visualization to support educators and researchers overwhelmed by large amounts of data generated by learning groups in computer mediated environments. Soller and colleague's (2005) review on support for collaborative learning summarizes key related work from the past decade. 
The Special IJAIEd Special Issue on CSCL (1998) was comprised of seven articles each of which cover more than one of the four ISLG dimensions discussed above. More recently, a series of workshops (Bader-Natal et al., 2010; Kim \& Kumar, 2012; Kumar \& Kim, 2013) held at the bi-annual AIEd \& ITS conferences have brought researchers interested in ISLG together. A total of 26 papers focussed on the ISLG were presented at these workshops. This special issue was conceived as a result of these workshops with the intent of attracting the wider AIEd community to this emerging field as well as archiving some of the existing high-quality research in the field of ISLG in the premier forum of the AIEd community.

\section{Contents of this Special Issue}

After two rounds of reviews and revisions, four of the twelve articles submitted to this special issue $(33 \%)$ were accepted. These four articles provide a sample of the field of ISLG. While the first article focuses on analysis of learner discourse to model groups, the other three articles apply similar techniques to provide real-time support to learning in groups.

Yoo and Kim (2014) address the problem of predicting learner performance in group settings using features observable in online learning environments such as content and frequency of messages posted by learners. The novel features used to track participation pattern of individuals and techniques used to aggregate heterogeneous classes of features are some of the key contributions of this work. The results and analysis presented offers insights that can shape current research in group assessment to supports educators as well as the learners themselves. The data set used in this work is collected over several semesters from college-level computer science courses. The courses employ a commonly used discussion forum to facilitate and capture asynchronous student discussion on course projects and assignments. The groups typically comprise of two or three students. However, the techniques and third-party tools used (most of which are publicly available) are easily applicable to other domains and learning settings. This article should serve as an excellent reference for researchers interested in applying contemporary AIEd techniques to the problem of modelling learning in groups.

Walker and colleagues (2014) extend their ongoing investigation on the use of adaptive support to improve the efficacy of peer-tutoring. This article takes a critical look at the existing evidence that has shown that adaptive support can be effective. They describe a well-designed experiment and conclusion drawn from it to tease apart the differences between real benefits of adaptive support and those that may be the result of increased learner motivation or engagement. This article offers a rare and interesting insight into the placebo effect that may be inadvertently caused by the novelty of use of advanced interactive technologies in educational applications. In terms of its ISLG characterization, this work describes a novel combination of simple text analytics with Bayesian knowledge tracing. Extensions of this technique offer promise for broader use in applications that deal with the challenge of student modelling using multiple noisy signals. Support is offered to learners playing the role of a peer-tutor to 
help them improve their help giving behaviours. As with the previous article, while this work is focused on dyads of high school students in an Algebra domain, the principles and techniques are applicable to other well-defined learning domains.

Pedagogical agents are a versatile technology for supporting learning in groups as evidenced by their use in three of the four articles included in this special issue. Specifically, agents capable of using natural language conversation to interact with learners can be customized to offer a variety of rich interactive support in online environments for synchronous group learning where the learners are often engaged in conversation among themselves. Tegos et al. (2014) presents MentorChat, a domainindependent system architecture for building group learning applications that are supported by conversational agents. One of the focuses of their work is the development of tools that allow educators to customize and control agent behaviour based on their own expert knowledge. In this article, their architecture is customized for a language learning application used by small groups of 2 to 3 students. Within this application domain, this article explores the use of directed prompts to elicit a higher degree of pedagogically beneficial responses from the learners. The form of directed prompts used here can be readily applied to other learning domains.

Adding to a decade long series of experiments on the use of conversational agents to support collaborative learning, the latest article by Rosé and colleagues focuses on delivering domain-independent interventions to promote academically productive collaboration among the learners. Similar to the previous article, learning groups are comprised of small homogenous groups (dyads and triads). Also, the authors present the Bazaar architecture. In contrast to MentorChat, this architecture focuses on agent development instead of end-to-end learning applications. The modular design of Bazaar allows these agents to be integrated with a variety of learning environments while being composed of a collection of reusable behavioural components. Unlike any of the other articles in this special issue, Adamson et al. (2014) discusses results from the use of similar support interventions across different Engineering and Science domains and learners age groups. This offers evidence of the maturity of ISLG research and indicates the state of readiness for their applicability outside the laboratory.

\section{Outlook}

Interaction between participants at the ISLG workshops $(2012,2013)$ led to discussion on several key research questions and future directions. In this section, we will summarize some of these directions.

\section{Modelling Learning in Groups}

Conventionally, learning in group is modelled as an aggregate function of the individual learning outcomes. However, this limits us to a macroscopic view of learning in groups and prohibits the study of the underlying processes. By capturing the finegrained observations of individual and group behaviour in state-of-the-art digital 
learning environments, we can decompose conventional models and study them at the granularity afforded by these observations. These techniques can be applied to visualizations such as group awareness widgets (Kreijns and Kirschner 2002), modelling group dynamics such as tracking stages of group development, prediction/ assessment of group performance and offering novel forms of support to learners.

\section{Optimal Group Formation}

Most of the existing CSCL \& ISLG research focuses on either ad-hoc groups or expertly crafted groups. Automatic optimal group formation is the task of forming groups given a pool of learners. This is a challenging problem because of the need to satisfy many pedagogical objectives and pragmatic constraints. The pedagogical objectives drive group composition in terms of distribution of learner skills, expertise and roles. Pragmatic constraints include scheduling and observing limits on group size. Multiple articles submitted to this special issue focused on this challenge from different perspectives.

Resources such as publicly available datasets that can help define metrics for optimal groups are required to advance the state-of-the-art on this challenge. Considering that online as well as classroom learning often includes group projects and encourages interaction among students, scalable approaches for capturing participants' needs and shaping groups can lead to fundamental contributions to the future of learning in groups.

\section{Managing Collaboration across Time and Space}

Existing work on learning in groups assumes homogenous technological affordances among learners. While this is a reasonable assumption for laboratory experiments where the learners are frequently collocated and use researcher specified computing devices, in real-life deployment scenarios, learners will use a wide variety of platforms that will render a spectrum of access capabilities and constraints. The challenge of seamlessly adapting to these variances across the members of a group is at the heart of successful scalability of ISLG.

\section{Tools that Support Educators}

The ultimate success of group learning will depend on the assimilation of these new methods into practice. However, educators (including teachers and administrators) generally have both limited time and limited cognizance of comprehensive assessment practices. Intelligent tools that dynamically process student activities, summarize both overall group work as well as individual student contributions and recommend best practices can support these stakeholders in maximizing the efficacy of learning in groups.

In conclusion, ISLG as a research field described in this preface offers opportunities to invent novel AIEd techniques as well as to apply well establish approaches to exciting problems. The four articles included in this special issue show a glimpse into these opportunities. We hope that the contents of this special issue will stimulate further 
interest in this field and motivate the readers to make their unique contributions towards shaping ISLG.

Acknowledgments This special issue was the result of concerted efforts by many individuals. Foremost, we would like to thank all the authors who submitted abstracts and papers for their contributions and patience throughout the review and publication process. We owe our gratitude to the 35 researchers who provided expert reviews for the papers submitted to this special issue. Their insightful feedback guided us through the peer-review process. Finally, a special thanks to the current editors in chief and their predecessors as well as the editorial staff of IJAIEd for their guidance and support from conception through the publication of this special issue.

\section{References}

Adamson, D., Dyke, G., Jang, H., \& Rosé, C. P. (2014). Towards an agile approach to adapting dynamic collaboration support to student needs. International Journal of Artificial Intelligence in Education, 24(1), 91-121. doi:10.1007/s40593-013-0012-6.

Bader-Natal, A., Walker, E., \& Rosé, C. (2010). Workshop on Opportunities for intelligent and adaptive behaviour in collaborative learning systems. In: Supplementary Proceedings of the Tenth International Conference on Intelligent Tutoring Systems, Pittsburgh, PA. URL: http://grockit.com/blog/itscollaboration-workshop/.

Baker, M. (1994). A model for negotiation in teaching-learning dialogues. International Journal of Artificial Intelligence in Education, 5(2), 199-254.

Barros, B., \& Verdejo, M. F. (2000). Analysing student interaction processes in order to improve collaboration: the DEGREE approach. International Journal of Artificial Intelligence in Education, 11(3), 221-241.

Bransford, J. D., Brown, A. L., \& Rodney, R. C. (2000). How people learn: Brain, mind, experience, and school. Washington, DC: National Academy Press.

Felder, R. M. (1995). A longitudinal study of engineering student performance and retention. IV. Instructional methods. Journal of Engineering Education, 84(4), 361-367.

Kim, J., \& Kumar, R. (2012). Proceedings of the full-day workshop on intelligent support for learning in groups. Held at the 11th International Conference on Intelligent Tutoring Systems (ITS 2012), Chania, Greece. URL: https://sites.google.com/site/islg2012/.

Kreijns, K., \& Kirschner, P. A. (2002). Group awareness widgets for enhancing social interaction in computersupported collaborative learning environments: Design and implementation. Conference on Frontiers in Education (FIE 2002), pp. 14-20.

Kumar, R., \& Kim, J. (2013). 2nd Workshop on Intelligent Support for Learning in Groups. In Proceedings of the Workshops at the 16th International Conference on Artificial Intelligence in Education (AIEd 2013), vol. 3, Memphis, TN. URL: https://sites.google.com/site/islg2013/.

Lave, J., \& Wenger, E. (1991). Situated learning. Legitimate peripheral participation. Cambridge: Cambridge University Press.

Soller, A., Martinez, A., Jermann, P., \& Muehlenbrock, M. (2005). From mirroring to guiding: a review of state of the art technology for supporting collaborative learning. International Journal of Artificial Intelligence in Education, 15(1), 324-331.

Special Issue on Computer Supported Collaborative Learning. (1998). International Journal of Artificial Intelligence in Education, 9(2).

Tegos, S., Demetriadis, S., \& Tsiatsos, T. (2014). A configurable conversational agent to trigger students' productive dialogue: a Pilot Study in the CALL domain. International Journal of Artificial Intelligence in Education, 24(1), 62-90. doi:10.1007/s40593-013-0007-3.

Vygotsky, L. (1978). Mind in society: The development of higher psychological processes. Cambridge: Harvard University Press.

Walker, E., Rummel, N., \& Keodinger, K. R. (2014). Adaptive intelligent support to improve peer tutoring in algebra. International Journal of Artificial Intelligence in Education, 24(1), 33-61. doi:10.1007/s40593013-0001-9.

Yoo, J., \& Kim, J. (2014). Can online discussion participation predict group project performance? Investigating the roles of linguistic features and participation patterns. International Journal of Artificial Intelligence in Education, 24(1), 8-32. doi:10.1007/s40593-013-0010-8 